\title{
Prognostic factors of IVF\&ICSI cycle cancellation in patients with endometriosis related infertility; A Retrospective Study.
}

\author{
Elif Kulahci Aslan ${ }^{1}$, Kiper Aslan ${ }^{2}$, Cihan Cakir ${ }^{1}$, Isil Kasapoglu ${ }^{1}$, Berrin Avci ${ }^{1}$, Baris Ata ${ }^{3}$, \\ and Gurkan Uncu ${ }^{4}$ \\ ${ }^{1}$ Uludag University Faculty of Medicine \\ ${ }^{2}$ Affiliation not available \\ ${ }^{3}$ Koc University School of Medicine \\ ${ }^{4}$ Uludag University School of Medicine
}

April 28, 2020

\begin{abstract}
Objective: To elucidate the prognostic factor for IVF\&ICSI cycle cancellation in patients with endometriosis related infertility Design \& Setting The study was a retrospective cohort study and conducted at Uludag University School of Medicine, ART center, between the years 2011-2017. Population Electronic database were screened and infertile patients with endometriosis, patients without male factor, systemic disease, undefined adnexal mass and aged $<40$ yrs were selected. Cycles were divided in two groups. (Cancelled vs. Embryo transferred) Results 44 cycles were cancelled and in 178 cycles embryo was able to transferred. When the groups were compared Age, day3 FSH were statically higher and AMH, AFC were statically lower in cycle cancelled (CC) group. Presence of adenomyosis was higher in CC group. (64\% vs. $40 \%$ p $<0.01)$ Surgery rate with laparotomy was higher in CC group. (54.5\% vs. $13.5 \% \mathrm{p}<0.01)$ AFC (OR:0.81, CI:0.70:0.93) remained as only independent factor associated with prognoses of IVF cycle with binary logistic regression analysis. Cancellation rates were similar between the phenotypes of endometriosis. Conclusions: Poor ovarian reserve, advanced age, presence of adenomyosis and the history of laparotomy are negative prognostic factors associated with IVF\&ICSI cycle cancellation in endometriosis related infertility. AFC is the only independent factor to predict cycle cancellation. Phenotype of endometriosis does not affect the results. There is no funding in this study. Key Words: Endometriosis, Infertility, Adenomyosis, Intracytoplasmic Sperm Injections,
\end{abstract}

\section{Title:}

Prognostic factors of IVF\&ICSI cycle cancellation in patients with endometriosis related infertility; A Retrospective Study.

\section{Running Title:}

Prognostic factors of Endometriosis \& Infertility

\section{Author Order}

1-Elif Kulahci ASLAN, M.D. a elifklhc@hotmail.com

2-Kiper ASLAN, M.D. a kiperaslan@yahoo.com.tr

3-Cihan CAKIR, M.D. M.Sc b cihancakir10@gmail.com

4-Isil KASAPOGLU, M.D. Assoc. Prof. a kasapogluisil@hotmail.com

5-Berrin AVCI, M.D. M.Sc. Assoc. Prof. b berrin@uludag.edu.tr 
6-Baris ATA, M.D. M.Sc. Prof. c barisata@hotmail.com

7-Gurkan UNCU M.D Prof. a guncu@gurkanuncu.org

\section{Department Affiliations}

a Uludag University School of Medicine Department of Obstetrics \& Gynecology Bursa, Turkey

b Uludag University School of Medicine Department of Histology \& Embryology Bursa, Turkey

c Koc University School of Medicine Department of Obstetrics \& Gynecology Istanbul, Turkey

\section{Corresponding Author:}

Kiper Aslan M.D. ${ }^{a}$

Görükle - Bursa / Turkey

kiperaslan@yahoo.com.tr Mobile: +905548127272

\section{Objective:}

To elucidate the prognostic factor for IVF\&ICSI cycle cancellation in patients with endometriosis related infertility

\section{Design \& Setting}

The study was a retrospective cohort study and conducted at Uludag University School of Medicine, ART center, between the years 2011-2017.

\section{Population}

Electronic database were screened and infertile patients with endometriosis, patients without male factor, systemic disease, undefined adnexal mass and aged $<40$ yrs were selected. Cycles were divided in two groups. (Cancelled vs. Embryo transferred)

\section{Results}

44 cycles were cancelled and in 178 cycles embryo was able to transferred. When the groups were compared Age, day3 FSH were statically higher and AMH, AFC were statically lower in cycle cancelled (CC) group. Presence of adenomyosis was higher in CC group. (64\% vs. $40 \% \mathrm{p}<0.01$ ) Surgery rate with laparotomy was higher in CC group. (54.5\% vs. $13.5 \% \mathrm{p}<0.01$ ) AFC (OR:0.81, CI:0.70:0.93) remained as only independent factor associated with prognoses of IVF cycle with binary logistic regression analysis. Cancellation rates were similar between the phenotypes of endometriosis.

\section{Conclusions:}

Poor ovarian reserve, advanced age, presence of adenomyosis and the history of laparotomy are negative prognostic factors associated with IVF\&ICSI cycle cancellation in endometriosis related infertility. AFC is the only independent factor to predict cycle cancellation. Phenotype of endometriosis does not affect the results.

\section{There is no funding in this study.}

Key Words: Endometriosis, Infertility, Adenomyosis, Intracytoplasmic Sperm Injections,

\section{Introduction}

Endometriosis is an inflammatory disorder and defined as presence of endometrial like tissue outside of the uterus and it's estimated prevalence is $10-15 \%$ in reproductive women.(1) It mainly induces infertility and prevalence of endometriosis rises above $40 \%$ in infertile women since endometriosis causes distortion of pelvic anatomy, impaired peritoneal function, altered implantation and decreased ovarian reserve. $(2-6)$ 
Treatment of endometriosis related infertility (ERI) is based on surgery, assisted reproductive technology (ART) or both of them. Assisted reproductive technologies may be ineffective in some cases with ERI since the all these factors listed above make the treatment more difficult. ICSI (intra cytoplasmic sperm injection) cycles frequently are being cancelled because of inefficient ovarian response to stimulation, low number or quality of collected oocytes(7), fertilization arrest or maturation arrest. In a study reported by Kuroda, it was found that cancellation rate of cycles (including cases of empty follicle, degenerating and unfertilized egg) were $35.5 \%$ in unoperated endometrioma, $47.1 \%$ in operated endometrioma, $40 \%$ in endometriosis with no endometrioma and $14.8 \%$ in tubal factor infertility.(8) Bongianni et. al. also found that cancellation rate was $7.5 \%$ in unoperated endometrioma, $9.8 \%$ in operated endometrioma and $2.9 \%$ in tubal factor infertility and commented as the cancellation rates are higher in ERI.(9) The reason of these high cancellation rates is still unclear. There is no study about whether which factors affect the cancellation rate in ERI.

In this study we aimed to elucidate the factors affecting the cancellation of cycle, depending on the phenotypes of endometriosis and other characteristics of the cycles.

\section{Material \& Method}

This was a retrospective cohort study. The study was conducted at Uludag University School of Medicine, ART center. The research ethics committee of Uludag University approved the study protocol (2017-1/52). Electronic database between Jan 2012 / Jan 2017 was screened to identify patients who have endometriosis.

Women aged $>40$ years, who had male infertility, chronic systemic diseases (diabetes mellitus, hypertension, etc.), and suspicion of malignancy were excluded from study. The enrolled patients' IVF\&ICSI cycles were compared in three endometriosis phenotypes; superficial endometriosis (SUP), ovarian endometrioma (OMA), deep infiltrating endometriosis(DE). If a patient had two phenotypes together, was enrolled in the more severe group. Superficial endometriosis was diagnosed during laparoscopic surgery, which has done for other indications like tubal factor infertility, unexplained infertility. It is defined as the presence of endometriotic lesions on the peritoneum. Ovarian endometrioma is defined as homogenous, usually unilocular, focal lesions in the ovary with low-level echoes in ultrasound imaging and with shading sign in magnetic resonance imaging.(10) DE was diagnosed by bimanual pelvic examination (palpable rectovaginal endometriotic nodule), by transvaginal ultrasound and MRI or diagnosed during endometriosis surgery as infiltrating type of endometriosis. $(11,12)$

Adenomyosis was defined as ingrowths of the endometrial cells into the myometrium. Patients were screened by transvaginal ultrasound and presence of two of the listed criteria was defined as adenomyosis. 1-No distinction of the endometrial-myometrial junction; 2-Asymmetry of the anterior and posterior myometrium; 3-Subendometrial myometrial striations; 4-Myometrial cysts and fibrosis; and 5-Heterogeneous myometrial echotexture. (13)

All patients were stimulated either by ultralong protocol, long protocol, antagonist protocol, micro-dose flare up or natural protocol. Goserelin acetate $3.6 \mathrm{mg}$ monthly depot form was injected for 3 months or daily leuprolide acetate $0.1 \mathrm{mg}$ / daily injection was applied for 3 months for pituitary desensitization in ultra-long protocol. Daily injections of leuprolide acetate were started on the 21th day of cycle in long protocol. After pituitary desensitization, daily gonadotropin injection with 150-600 IU/day was started and daily antagonist (certrorelix-ganirelix $0.25 \mathrm{mg}$ ) injections were applied after reaching 12-14 mm follicle in short antagonist protocol. Trigger (recombinant hCG or Leuprolide acetate) was given when $>3$ follicles greater than $17 \mathrm{~mm}$. Oocyte retrieval was done 34-36 hours after hCG injection. Luteal phase was supported with vaginal $\% 8$ progesterone gel, two times a day, starting on the evening of oocyte retrieval and continued until a negative pregnancy test or detection of fetal cardiac activity. Day of embryo transfer was decided based on the number of available embryos, embryo quality.

Three types of cycle cancellation were defined.

Type A is identified as no follicular development despite the maximum dose of gonadotropin injections.

Type B is identified as full fertilization failure. 
Type $\mathrm{C}$ is identified as maturation arrest and is defined whenever the maturation of embryo stopped. (Table $-1)$

All cycles were compared as 2 groups. First group was consisted of cancelled cycles (CC); the second group was consisted of the embryo transferred (ET) cycles. Both of the groups were divided in three subgroups depending on the phenotype of endometriosis. Factors associated with cycle cancellation depending on the phenotype of the disease were found. We further investigated independent risk factors associated with cycle cancellation with logistic regression analysis. Also we analyzed the effects of presence of adenomyosis and laparoscopy or laparotomy on ovarian reserve.

Kolmogorov Smirnov test was used for assessing whether the variables follow a normal distribution or not. Variables were reported as mean \pm standard and median (minimum:maximum) deviation values. According to the normality test result, independent samples t-test was used for between-group comparisons. Categorical variables were reported as n(\%) and compared by Pearson Chi-square test or Fisher-Freeman-Halton-Test. In order to determine the independent risk factors that affect IVF cycle cancellation, binary logistic regression analysis with backward selection was performed. SPSS (IBM Corp. Released 2012. IBM SPSS Statistics for Windows, Version 21.0. Armonk, NY: IBM Corp.) was used for performing statistical analysis and the level of significance was set at $\alpha=0.05$.

Results

Between the years Jan 2012- Jan 2017 there were 2515 cycles. All cycles were screened and 222 of the cycles were enrolled in the study. Demographic data of 222 cycles is presented in Table-2. Median age was 31 (20 - 40) years. Median duration of infertility was 4 (1 -18) years.

There were 18 (8.1\%) patients with superficial endometriosis, 157 (70.7\%) patients with ovarian endometrioma and $47(21.2 \%)$ patients with deep infiltrating endometriosis.

The presence of history of surgery was $56.7 \%$ as $20.6 \%$ of was by laparotomy and $79.4 \%$, by laparoscopy.

Median day $3 \mathrm{FSH}$ was 6.7 (2.6 - 23.2) IU/dl, median day $3 \mathrm{E}_{2}$ was 43 (15 -310) pg/ml and median AMH was $1.9(0.1-10) \mathrm{ng} / \mathrm{ml}$. Median AFC was 7 (1 - 20)

Overall live birth rate was $18 \%$ per cycle and $24.6 \%$ per embryo transfer. (Table-4)

$44(19.8 \%)$ cycles were cancelled and in $178(80.2 \%)$ cycles embryo was able to transferred. (Table-3)

$4(9.1 \%)$ cycles due to inefficient response (Type A), 24 (54.6\%) cycles due to fertilization failure (Type B) and $16(36.3 \%)$ cycles due to maturation arrest (Type C) were cancelled.

When the groups were compared, age was higher in CC group. $(34(26-43)$ yrs. vs. $30(8-40)$ yrs. $\mathrm{p}<0.01)$

Day 3 FSH levels were higher in CC group., respectively $(8.1(2.6-23.2) \mathrm{IU} / \mathrm{dl}$ vs. $6.2(2.6-21.8) \mathrm{IU} / \mathrm{dl}$ $\mathrm{p}<0,01)$

AMH level was lower in CC group. $(0.5(0.01-10) \mathrm{ng} / \mathrm{ml}$ vs. $1.9(0.01-10) \mathrm{ng} / \mathrm{ml} \mathrm{p}<0.01)$

AFC was lower in CC group. $(4(1-16)$ vs. $8(1-20) \mathrm{p}<0.01)$

Presence of adenomyosis was higher in CC group. (64\% vs. $40 \% \mathrm{p}<0.01$ )

Surgery rate with laparotomy was higher in CC group. (54.5\% vs. $13.5 \%$ p $<0.01)$ (Table-5)

We further analyzed the statistically significantly different factors $(\mathrm{p}<0.05)$ with binary logistic regression analysis to determine the independent risk factors that are prognostic for cycle cancellation. AFC (OR:0.81, CI:0.70:0.93) remained as the only independent factor associated with prognoses of IVF cycle. (Table-6)

We analyzed the presence of adenomyosis and presence of surgery history with laparotomy vs. laparoscopy to find whether has negative impact on ovarian reserve. AFC was lower in patients with adenomyosis. (8 
$(1-20)$ vs $6(1-16), \mathrm{p}<0.01)$ But other ovarian reserve markers (day3 FSH, day3 E2, AMH) were similar in patients with / without adenomyosis. (Table - 9) AFC and AMH levels were lower in patients with laparotomy history than the patients with laparoscopy history. (AFC; $4(1-9)$ vs $7(1-20), \mathrm{p}<0.01-$ AMH; $0.2(0.1-10)$ vs $1.72(0.1-5.2)$, p:0.02) (Table-10)

Cancellation rates were as $16.6 \%$ in SUP, $19.7 \%$ in OMA, $21.2 \%$ in DE and there was no statistically significant differences between the phenotypes of endometriosis. (Table-7)

Discussion

It's largely known that endometriosis has challenging impact on infertility treatment. Both, couples and doctors are starting the infertility treatment with high hopes but as our results, $20 \%$ of couples with endometriosis related infertility (ERI) are knocked down in this battle because of cycle cancellation.

In this study we aimed to elucidate whether the phenotype of endometriosis affects the number of cancelled cycles, and to obtain the factors related with cancelled cycles. Our findings clearly indicated that phenotype of endometriosis does not affect the success of cycles and only ovarian reserve markers, particularly AFC, have prognostic value to predict the risk of cancelling the cycle.

The cycle cancellation rate (CCR) of all ERI cycles was $19.8 \%$ in our study. There are limited numbers of studies, which inform about cycle cancellation rates of ERI. All of these studies have different CC rates. CC rates of studies in the literature are variable between $7.5 \%$ and $47.1 \% .(8,9,14)$ Our CC results are $16.6 \%$ in SUP, $19.7 \%$ in OMA and $21.2 \%$ in DE group.

According to our results, we found four prognostic factors related with CC. These factors are age, ovarian reserve (day3 FSH, AMH and AFC), adenomyosis rate and laparotomy rate. With logistic regression analysis, antral follicle count is the only independent factor related with cycle cancellation.

Age

It's largely known that advance age negatively affects fertility. As age increases, fecundity decreases. The fecundity of women decreases gradually after 32 years but it significantly decreases after 37 years.(15) The reason of age related decreasing fertility may because of altered ovarian reserve and metabolism. In a prospective study about women age and ovarian reserve, altered follicular metabolism was found in women with either reduced ovarian reserve or advanced maternal age. (16)

Presence of endometriosis has also detrimental effect to ovarian reserve by itself. Our ERROR (Endometrioma Related Reduction in Ovarian Reserve) study showed that women with endometrioma experience a progressive decline in serum AMH level, which is faster than in healthy women. Thus, both presence of endometriosis and advanced age may have negative impact on fertility. It may be related with cycle cancellation. In our study we found mean women age $34(26-43)$ in CC and $30(8-40)$ in ETC (embryo transferred cycle) group. According to our results, women age is one of the prognostic factors for predicting CC.

\section{Ovarian Reserve}

We found increased day 3 FSH and AMH level and decreased AFC level in CC group. All these factors are related with ovarian reserve. Also AFC was found as the only independent factor related with cycle cancellation. These findings clearly indicated that decreased ovarian reserve, especially decreased AFC is related with high possibility of cycle cancellation independent of endometriosis subtypes.

There is a study analyzing the poor response and cycle cancellations according to AMH levels during different days of menstrual cycle. The results indicated that day3 AMH level below $0.3 \mathrm{ng} / \mathrm{ml}$ predicts high risk of CC with $70 \%$ sensitivity and $90 \%$ specificity. In our study we found AMH level $0.5(0.01-10) \mathrm{ng} / \mathrm{ml}$ in CC group. (It was $1.9(0.01-10) \mathrm{ng} / \mathrm{ml}$ in ETC group) All these findings indicated that poor ovarian reserve is related with increased risk of CC in ERI. 
It is clearly known that AFC is a good predictor of ovarian response even in ovary with endometrioma.. In our study AFC was found the only independent prognostic factor related with CC. (OR:0.81, CI:0.70:0.93) This result shows the importance of AFC for assessing ovarian reserve and to predict cycle cancellation.

Previously in our prospective ERROR (Endometrioma Related Reduction in Ovarian Reserve) study, we evaluated that endometrioma is associated with progressive decline in ovarian reserve than normal healthy women.(17) In another study done in our department related to oocyte quality in endometriosis showed that endometriosis negatively affects the oocyte morphology but not pregnancy rates. In this study, patients were divided in two groups, as male factor and endometriosis. Of the abnormal oocytes determined in all cycles, $59.1 \%$ of oocytes were in endometriosis group and $40.9 \%$ of oocytes in male factor group. The difference was statically significance. (7)

In our study, we formed a new classification for cycle cancellation. Type A ; inefficient ovarian response, Type B; fertilization failure, Type C; maturation arrest. As our results, we found CCR $9.1 \%$ as Type A, 54,6\% as Type B, 36,3\% as Type C. We may say that the main reason of cancellation is the fertilization problem with $54 \%$. The results show that presence of endometriosis negatively effects ovarian quality while decreasing ovarian reserve. And if a patient with endometriosis has a poor ovarian reserve, the risk of cycle cancellation is higher than the ones with normal ovarian reserve independently of the subtypes of endometriosis

\section{History of Laparotomy}

Another prognostic factor found is presence of history of laparotomy. In our results, laparotomy rate was $54.5 \%$ in CC group and $13.5 \%$ in ETC group. $(\mathrm{p}<0.01)$ and the difference was statistically significant. It's an ongoing debate whether laparotomy decreases ovarian reserve. There are studies about surgery in endometriosis and results indicate that laparoscopy may have more detrimental effects on ovarian reserve than laparotomy.(18) But adversely; our results indicated that the ovarian reserve was worse in the patients whom operated by laparotomy than laparoscopy. (Table -10) We didn't know the ovarian reserve of the patients before surgery, so we couldn't interpret the effect of surgery type on ovarian reserve. Furthermore we didn't know whether stripping or coagulation were used during endometrioma excision. As the result we may conclude that (excluding contributing factors) presence of laparotomy history has negative impact on ovarian reserve.

\section{Adenomyosis}

The other issue concerning cancellation is the presence of adenomyosis. We found adenomyosis rate $64 \%$ (28/44) in CC group and 40\% (16/44) ETC group and the difference was statistically significant

Nevertheless, it was found that the presence of adenomyosis is not an independent marker of cycle cancellation in the univariate analysis.

Although it is not an independent factor in terms of cancellation, we can say that it is important to determine the presence of adenomyosis in terms of cancellation since it was shown that the AFC is worse in the presence of adenomyosis $(8(1-20)$ vs. $6(1-16), \mathrm{p}<0.01)$. This result may be strengthened with larger sample size.

There were 28 cancelled cycles with adenomyosis in our study. $3(10.7 \%)$ of these cycles were cancelled because of inefficient ovarian response, $16(57 \%)$ of these cycles cancelled because of fertilization problem and $9(32 \%)$ of these cycles cancelled because of maturation problem. (Table- 8 )

Although it is not possible to make statistical analysis due to the small number of cases, it is thought that more than half of the cancellation cases in the presence of adenomyosis have insufficient ovarian response and this may be related to ovarian reserve. There is no data in the literature about how ovarian reserve is affected in the presence of adenomyosis.

Beyond of all these factors discussed above, we also analyzed whether endometriosis phenotype is predictive for cycle cancellation. In our study it was found that the cycle cancellation is mainly based on ovarian functions and endometriosis phenotype does not affect the cycle cancellation rates.. Presence of adenomyosis and laparotomy history are also negative factors both on ovarian reserve and cycle cancellation. 
We can predict the risk of cancellation with analyzing ovarian reserve parameters carefully. Day3 FSH, $\mathrm{AMH}, \mathrm{AFC}$ are important parameters to predict cycle success in ERI. AFC is the only independent factor to predict cycle success. Results can be confirmed with further larger scale studies.

\section{Declarations:}

Acknowledgments:

None

Funding:

There is no funding in this study.

Conflict of Interest:

There is no conflict of interest in this study.

Contribution to Authorship:

Elif Külahçı Aslan Collecting Data, Analysis, Writing

Kiper Aslan Analysis, Writing

Cihan Cakir Embryology Procedures

Isil Kasapoglu Folliculometry, Oocyte-Pick Up, Embryo Transfer Procedures

Berrin Avci. Embryology Procedures

Baris Ata. İnterpreting the results, Reviewing

Gürkan Uncu İnterpreting The results, Reviewing

Ethic Approval:

The research ethics committee of Uludag University approved the study protocol with the approval number 2017-1/52 on 07.02.17.

\section{References}

1. Mowers EL, Lim CS, Skinner B, Mahnert N, Kamdar N, Morgan DM, et al. Prevalence of Endometriosis During Abdominal or Laparoscopic Hysterectomy for Chronic Pelvic Pain. Obstet Gynecol [Internet]. 2016 [cited 2019 Aug 28];127(6):1045-53. Available from: http://www.ncbi.nlm.nih.gov/pubmed/27159755

2. Eskenazi B, Warner ML. Epidemiology of endometriosis. Obstet Gynecol Clin North Am [Internet]. 1997 Jun [cited 2019 Aug 28];24(2):235-58. Available from: http://www.ncbi.nlm.nih.gov/pubmed/9163765

3. Lebovic DI, Mueller MD, Taylor RN. Immunobiology of endometriosis. Fertil Steril [Internet]. 2001 Jan [cited 2019 Aug 28];75(1):1-10. Available from: https://linkinghub.elsevier.com/retrieve/pii/S0015028200016307

4. Kao LC, Germeyer A, Tulac S, Lobo S, Yang JP, Taylor RN, et al. Expression profiling of endometrium from women with endometriosis reveals candidate genes for disease. Based implantation failure and infertility. Endocrinology. 2003 Jul 1;144(7):2870-81.

5. Endometriosis and infertility: A committee opinion. Fertil Steril. 2012;98(3):591-8.

6. Uncu G, Kasapoglu I, Ozerkan K, Seyhan A, Oral Yilmaztepe A, Ata B. Prospective assessment of the impact of endometriomas and their removal on ovarian reserve and determinants of the rate of decline in ovarian reserve. Hum Reprod. 2013;28(8):2140-5.

7. Kasapoglu I, Kuspinar G, Saribal S, Turk P, Avcı B, Uncu G. Detrimental effects of endometriosis on oocyte morphology in intracytoplasmic sperm injection cycles: a retrospective cohort study. Gynecol Endocrinol. 2018 Mar 4;34(3):206-11. 
8. Kuroda K, Kitade M, Kikuchi I, Kumakiri J, Matsuoka S, Kuroda M, et al. The impact of endometriosis, endometrioma and ovarian cystectomy on assisted reproductive technology. Reprod Med Biol. 2009;8(3):1138.

9. Bongioanni F, Revelli A, Gennarelli G, Guidetti D, Delle Piane LD, Holte J. Ovarian endometriomas and IVF: a retrospective case-control study. Reprod Biol Endocrinol [Internet]. 2011 Jun 17 [cited 2019 Aug 28];9:81. Available from: http://www.ncbi.nlm.nih.gov/pubmed/21679474

10. Glanc P, Benacerraf B, Bourne T, Brown D, Coleman BG, Crum C, et al. First International Consensus Report on Adnexal Masses: Management Recommendations. J Ultrasound Med. 2017 May 1;36(5):849-63.

11. Woodward PJ, Sohaey R, Mezzetti TP. Endometriosis: radiologic-pathologic correlation. Radiographics [Internet]. [cited 2019 Aug 28];21(1):193-216; questionnaire 288-94. Available from: http://www.ncbi.nlm.nih.gov/pubmed/1

12. De Cicco C, Corona R, Schonman R, Mailova K, Ussia A, Koninckx PR. Bowel resection for deep endometriosis: A systematic review. Vol. 118, BJOG: An International Journal of Obstetrics and Gynaecology. 2011. p. 285-91.

13. Bazot M, Daraï E, Rouger J, Detchev R, Cortez A, Uzan S. Limitations of transvaginal sonography for the diagnosis of adenomyosis, with histopathological correlation. Ultrasound Obstet Gynecol [Internet]. 2002 Dec [cited 2019 Aug 28];20(6):605-11. Available from: http://www.ncbi.nlm.nih.gov/pubmed/12493051

14. Garcia-Velasco JA, Mahutte NG, Corona J, Zúñiga V, Gilés J, Arici A, et al. Removal of endometriomas before in vitro fertilization does not improve fertility outcomes: a matched, case-control study. Fertil Steril [Internet]. 2004 May [cited 2019 Aug 28];81(5):1194-7. Available from: http://www.ncbi.nlm.nih.gov/pubmed/15136074

15. American College of Obstetricians and Gynecologists Committee on Gynecologic Practice and The Practice Committee of the American Society for Reproductive Medicine. Female age-related fertility decline: Cmmitee Opinion No. 589. Fertil Steril [Internet]. 2014 [cited 2019 Aug 28];101(3):633-4. Available from: http://dx.doi.org/10.1016/j.fertnstert.2013.12.032

16. Pacella L, Zander-Fox DL, Armstrong DT, Lane M. Women with reduced ovarian reserve or advanced maternal age have an altered follicular environment. Fertil Steril [Internet]. 2012 Oct [cited 2019 Aug 28];98(4):986-94.e1-2. Available from: http://www.ncbi.nlm.nih.gov/pubmed/22818290

17. Kasapoglu I, Ata B, Uyaniklar O, Seyhan A, Orhan A, Yildiz Oguz S, et al. Endometrioma-related reduction in ovarian reserve (ERROR): a prospective longitudinal study. Fertil Steril. 2018 Jul 1;110(1):1227 .

18. Zaitoun MM, Zaitoun MM, El Behery MM. Comparing long term impact on ovarian reserve between laparoscopic ovarian cystectomy and open laprotomy for ovarian endometrioma. J Ovarian Res. 2013;6(1).

Tables

Table -1 Cycle Cancellation Types

\begin{tabular}{ll}
\hline Cycle Cancellation Types & Reasons of Cancellation \\
\hline Type A & Inefficient Ovarian Response \\
Type B & Fertilization Failure \\
Type C & Maturation Arrest \\
\hline
\end{tabular}

Table -2 Demographic data of all cycles

\begin{tabular}{ll}
\hline & $\mathrm{n}: 222$ \\
\hline Age & $31(20-40)$ \\
BMI $\left(\mathrm{kg} / \mathrm{m}^{2}\right)$ & $23.4(16-37)$
\end{tabular}




\begin{tabular}{ll}
\hline & $\mathrm{n}: 222$ \\
\hline Duration of infertility (yrs) & $4(1-18)$ \\
Gravida $012>3$ & $169(76,1 \%) 44(19.9 \%) 5(2.2 \%) 4(1$ \\
Parity $01>2$ & $217(97.7 \%) 5(2.3 \%) 0$ \\
Infertility Type Primary Secondary & $169(76.1 \%) 53(23.9 \%)$ \\
Endometriosis Phenotype * Superficial * OMA * DE *OMA (+) *OMA $(-)$ & $18(8.1 \%) 157(70.7 \%) 47(21.2 \%) * 3$ \\
History of Surgery Laparotomy Laparoscopy & $126(56.7 \%) 26(20.6 \%) 100(79.4 \%)$ \\
Surgery Type ; OMA Bilateral Unilateral DE SUP & $73 / 126(57.9 \%) 13 / 126(10.3 \%) 60 / 12$ \\
Ovarian Reserve Day3 FSH (IU/dl) Day3 Estradiol $(\mathrm{pg} / \mathrm{ml})$ AFC (n) AMH $(\mathrm{ng} / \mathrm{ml})$ & $6.7(2.6-23.2) 43(15-310) 7(1-$ \\
\hline
\end{tabular}

Note: Values are medians with minimum and maximum

Table- 3 Treatment Parameters of all cycles.

No. Of Previous Cycle 12345

Stimulation Protocols Antagonist Ultralong Long Natural Microdose Flare Up

Duration of Stimulation

Total Gonadotropin Dosage (IU) *

Estradiol (pg/ml)(day of hCG)

Cycles Cancelled Inefficient Ovarian Response Fertilisation Failure Maturation Arrest
$222135(60.8 \%) 60(27 \%) 16(7.2 \%$

$22298(44.4 \%) 83(37.3 \%) 32(14.3$

$10(1-17)$

$3050+1087$

$881(31-5282)$

$44 / 222(\% 19.8) 4 / 44(9.1 \%) 24 / 44$

* Note: mean value with standart deviation

Table -4 Pregnancy Outcomes of all cycles.

\begin{tabular}{ll}
\hline No. of picked up oocyte & $8(0-35)$ \\
\hline No. of MII oocyte & $5(0-26)$ \\
No. of 2PN oocyte & $3(0-15)$ \\
Positive B-hCG (per cycle) & $66 / 222(29.7 \%)$ \\
Positive B-hCG (per transfer) & $66 / 165(40 \%)$ \\
Biochemical abort (per cycle) & $17 / 222(7.6 \%)$ \\
Anembryonic pregnancy rate (per cycle) & $7 / 222(3.1 \%)$ \\
Clinical pregnancy rate* (per cycle) & $42 / 222(18.9 \%)$ \\
Clinical pregnancy rate* (per transfer) & $42 / 165(25.4 \%)$ \\
İmplantasyon Oranı (Embriyo Başına) & $52 / 262(20 \%)$ \\
Missed abortus rate (per cycle) & $2 / 222(1 \%)$ \\
Live birth rate (per cycle) & $40 / 222(18 \%)$ \\
Live birth rate (per transfer) & $40 / 165(24.6 \%)$ \\
\hline
\end{tabular}

Note: Values are medians with minimum and maximum

Table-5 Cancelled cycles vs. Transferred cycles - Demographic Data

\begin{tabular}{llll}
\hline & Cancelled(CC) $(\mathrm{n}: 44)$ & $\begin{array}{l}\text { Transferred(ET) } \\
(\mathrm{n}: 178)\end{array}$ & $\mathrm{p}$ \\
\hline Age & $34(26-40)$ & $30(8-40)$ & $<\mathbf{0 . 0 1}$ \\
BMI $\left(\mathrm{kg} / \mathrm{m}^{2}\right)$ & $23.4(18.9-36.1)$ & $23.3(16-37)$ & 0.313 \\
Gravida & $0(0-2)$ & $0(0-4)$ & 0.729
\end{tabular}




\begin{tabular}{llll}
\hline & Cancelled(CC) $(\mathrm{n}: 44)$ & $\begin{array}{l}\text { Transferred(ET) } \\
(\mathrm{n}: 178)\end{array}$ & $\mathrm{p}$ \\
\hline $\begin{array}{l}\text { Duration of infertiliy } \\
\text { (yrs) }\end{array}$ & $5(1-18)$ & $4(1-16)$ & 0.754 \\
Day3 FSH(IU/dl) & $8.1(2.6-23.2)$ & $6.2(2.6-21.8)$ & $<\mathbf{0 . 0 1}$ \\
Day3 Estradiol $(\mathrm{pg} / \mathrm{ml})$ & $46.1(5.4-240)$ & $42(1.5-310)$ & 0.07 \\
AMH(ng/ml) & $0.5(0.01-10)$ & $1.9(0.01-10)$ & $<\mathbf{0 . 0 1}$ \\
AFC (n) & $4(1-16)$ & $8(1-20)$ & $<\mathbf{0 . 0 1}$ \\
Adenomyozis(\%) & $28 / 44(\% 64 \%)$ & $72 / 178(\% 40 \%)$ & $<\mathbf{0 . 0 1}$ \\
Surgery (\%) & $22 / 44(\% 50 \%)$ & $104 / 178(\% 58 \%)$ & 0.255 \\
Surgery ; Laparoscopy & $10 / 22(45.5 \%) 12 / 22$ & $90 / 104(86.5 \%) 14 / 104$ & $<\mathbf{0 . 0 1}$ \\
Laparotomy & $(54.5 \%)$ & $(13.5 \%)$ & \\
Endometriosis Phenotype & $3 / 44(6.8 \%) 31 / 44$ & $15 / 178(8.4 \%) 126 / 178$ & 0.916 \\
SUP OMA DE & $(70.5 \%) 10 / 44(22.7 \%)$ & $(70.8 \%) 37 / 178(20.8 \%)$ & \\
\hline
\end{tabular}

Note: Values are medians with minimum and maximum

Table- 6 Significant prognostic factors of cycle cancellation after logistic regression analysis

\begin{tabular}{llll}
\hline Factors & Wald & OR $(95 \% \mathrm{CI})$ & $\mathrm{p}$ \\
\hline AFC & 8.70 & $\mathbf{0 . 8 1}(0.70: 0.93)$ & 0.003 \\
Model $\chi^{2}=16.55 ; \mathrm{p}<0.001$ & Model $\chi^{2}=16.55 ; \mathrm{p}<0.001$ & Model $\chi^{2}=16.55 ; \mathrm{p}<0.001$ & Model $\chi^{2}=16.55 ; \mathrm{p}<0.001$ \\
Pseudo $\mathrm{R}^{2}=0.16$ & Pseudo $\mathrm{R}^{2}=0.16$ & Pseudo $\mathrm{R}^{2}=0.16$ & Pseudo $\mathrm{R}^{2}=0.16$ \\
$\mathrm{n}=222$ & $\mathrm{n}=222$ & $\mathrm{n}=222$ & $\mathrm{n}=222$ \\
\hline
\end{tabular}

OR: Odds ratio, CI:Confidence Interval

Table-7 Cycle Cancellation - Depending on Phenotypes of endometriosis

\begin{tabular}{|c|c|c|c|c|c|}
\hline & Superficial & Endometrioma & $\begin{array}{l}\text { Deep } \\
\text { Infiltrating }\end{array}$ & Total & $\mathrm{p}$ \\
\hline $\begin{array}{l}\text { No. of } \\
\text { cancelled } \\
\text { cycles }\end{array}$ & $3 / 18(16.6 \%)$ & $31 / 157(19.7 \%)$ & $10 / 47(21.2 \%)$ & $44 / 22219.8 \%$ & $0.916^{*}$ \\
\hline $\begin{array}{l}\text { Inefficient } \\
\text { response }\end{array}$ & $0 / 18$ & $1 / 157(0.6 \%)$ & $3 / 47(6.3 \%)$ & $4 / 449 \%$ & $0.147^{* *}$ \\
\hline $\begin{array}{l}\text { Fertilization } \\
\text { Failure }\end{array}$ & $2 / 18(11.1 \%)$ & $17 / 157(10.8 \%)$ & $5 / 47(10.6 \%)$ & $24 / 4454 \%$ & \\
\hline $\begin{array}{l}\text { Maturation } \\
\text { Arrest }\end{array}$ & $1(5.5 \%)$ & $13 / 157(8.2 \%)$ & $2 / 47(4.2 \%)$ & $16 / 4436 \%$ & \\
\hline
\end{tabular}

${ }^{*}$ kruskal wallis , ${ }^{* *}$ Fisher - Freeman Halton Test.

Table- 8 Patients with adenomyosis (divided subtypes) - Cycle Cancelled

\begin{tabular}{lllll}
\hline & Inefficient Response & Fertilization Failure & Maturation Arrest & Total \\
\hline Superficial & 0 & 2 & 1 & 3 \\
Ovarian Endometrioma & 0 & 10 & 6 & 16
\end{tabular}




\begin{tabular}{lllll}
\hline & Inefficient Response & Fertilization Failure & Maturation Arrest & Total \\
\hline Deep İnfiltrating & 3 & 4 & 2 & 9 \\
Total & 3 & 16 & 9 & 28 \\
\hline
\end{tabular}

Table - 9 Ovarian Reserve \& Presence of Adenomyosis

\begin{tabular}{llll}
\hline & Adenomyosis ( - ) (n: 122) & Adenomyosis ( + ) (n: 100) & $\mathrm{p}$ \\
Day3 FSH & $6.7(2.6-23.2)$ & $6.4(2.6-19.6)$ & 0.360 \\
Day3 E2 & $44.9(15-159)$ & $42.4(14-310)$ & 0.971 \\
AFC & $8(1-20)$ & $6(1-16)$ & $<\mathbf{0 . 0 1}$ \\
AMH & $1.9(0.1-10)$ & $1.67(0.1-10)$ & 0.09 \\
\hline
\end{tabular}

Table -10 Ovarian Reserve \& Laparotomy vs Laparoscopy

\begin{tabular}{llll}
\hline & Laparotomy (n:26) & Laparoscopy $(\mathrm{n}: 100)$ & $\mathrm{p}$ \\
Day3 FSH & $7.3(2.6-14.4)$ & $6.3(3-19)$ & 0.206 \\
Day3 E2 & $57(20-119)$ & $42(7.2-310)$ & 0.08 \\
AFC & $4(1-9)$ & $7(1-20)$ & $<\mathbf{0 . 0 1}$ \\
AMH & $0.2(0.1-10)$ & $1.72(0.1-5.2)$ & $\mathbf{0 . 0 2}$ \\
\hline
\end{tabular}

Note: Values are medians with minimum and maximum 